\title{
Positive Observers for Positive Interval Linear Discrete-Time Delay Systems
}

\author{
Ping Li James Lam Zhan Shu
}

\begin{abstract}
Linear matrix inequalities (LMIs) provide a powerful analysis and synthesis framework for linear systems. In this paper, we use LMIs to develop positive observers for positive linear discrete-time (PLDT) systems with both parameter uncertainties and time delay. Specifically, we first present some equivalent conditions for the asymptotic stability of positive linear discrete-time delay systems, which will be employed to design the positive observers. Then, necessary and sufficient conditions are proposed to check the existence of positive observers for interval PLDT systems with time delay when the positivity of the error signals is considered, and the observer matrices to be constructed can be easily obtained through the solutions of LMIs. Finally, a numerical example is provided to demonstrate the efficacy of the proposed approach.
\end{abstract}

\section{INTRODUCTION}

In many practical systems, variables are constrained to be positive. Such constraints naturally arise in physical systems where variables are used to represent concentrations of material, population numbers of bacteria or cells or, in general, measures. Examples include pollutant transport, chemotaxis, pharmacokinetics, industrial engineering involving chemical reactors, heat exchangers, storage systems. These systems are commonly referred to as positive systems, whose state variables and outputs are constrained to be positive (or at least nonnegative) in value at all times whenever the initial condition and input are nonnegative. The mathematical theory of positive systems is based on the theory of nonnegative matrices founded by Perron and Frobenius. For references, we refer readers to [1], [2].

Due to the wide range of applications of positive systems, it is important to study their analysis and synthesis problems. However, the positivity of the system state will bring about many new issues, which cannot be simply solved by using well-established results for general linear systems, since positive systems are defined on convex cones rather than linear spaces. For instance, similarity transformation, which plays an important role in exploring the property of general linear systems, may not be applicable for positive systems in general due to the positivity constraints on system matrices. Therefore, problems of positive systems have attracted a lot of attention from researchers all over the world, and many

This work was partially supported by RGC HKU 7031/07P, and by Science Foundation of Ireland, Grant 07/PI/I1838

P. Li and J. Lam are with the Department of Mechanical Engineering, The University of Hong Kong, Pokfulam Road, Hong Kong. pingli@hkusua.hku.hk and james. lam@hku.hk

Z. Shu was with the Department of Mechanical Engineering, The University of Hong Kong, and is now with the Hamilton Institute, National University of Ireland, Maynooth. hustd8@gmail . com fundamental results have been reported recently [3] [4] [5] [6] [7] [8] [9] [10] [11] [12]. To mention a few, the stability issue of positive systems has been treated in [4] [7] [13], and the positive realization problem has been extensively studied in the past few years, see the tutorial paper [8] and references therein. Reachability and controllability for positive systems can be found in [3] and [9]. In terms of linear matrix inequality (LMI) approach and linear programming techniques, the synthesis problem of controllers ensuring the stability and positivity of the closed-loop systems has been proposed in [10] and [11], respectively.

In contrast with the abundance in the property characterization, and stabilization, little attention has been paid to the positive observer design problem. The first study of such subject is initiated in [6], where a structural decomposition approach has been presented to design the positive observers for compartmental systems. In [12], both the Sylvester equation approach and the positive realization approach are proposed to study the existence and synthesis of positive observers of positive linear systems. It should be emphasized that most aforementioned results shared a severe restriction that system parameters should be known exactly such that some nice techniques, like matrix decomposition and coordinates transformation, can be applied to construct the desired observers. However, in practice, it is unavoidable that there exist uncertainties due to the limitation in parameter acquisition and errors in measurement. Although some results on robust stability of positive systems are available in [4], the existence and design scheme of positive observers for positive systems have not been fully investigated, see [14].

In addition, a key limitation of modeling the positive compartmental systems is that material transfers among different compartments are not instantaneous, which further implies that time delays should be accounted for when capturing the realistic dynamics of these systems. On one hand, it has been well known that, for general linear systems, delay plays a crucial role in the stability performances, and may be the source of some complicated behavior, such as instability, oscillation, and even chaos. On the other hand, it is shown that the presence of time delays does not affect the stability performance of positive linear systems, see [7], [15]. In [16], an identical time-delay (Luenberger-type) observer is proposed to estimate the state of positive time-delay systems, where the observer should be designed based upon the original system with exactly known parameters. Note that, as we mentioned before, such an approach cannot be applied to the systems with parameter uncertainties any more. Thus, how to study the synthesis of positive observers for positive 
systems in the presence of both parameter uncertainties and time delay is still an open question; moreover, how can we access the effect of time delays on the synthesis of positive observers? These constitute the main focuses of the present paper.

Based on the above discussion, in this paper, we are concerned with the positive observer design for positive linear discrete-time systems with both interval parameter uncertainties and time delay. To achieve this, we first present some equivalent conditions to guarantee the stability of positive discrete-time systems with time delay. Then, we develop a novel characterization for the positive observer synthesis in terms of vertical matrices of the uncertain domain which system matrices belong to. More specifically, we provide not only necessary and sufficient conditions to check the existence of positive observers for interval PLDT systems with time delay, but also an easily computable approach to construct the observer matrices. It should be noted that these conditions are independent of time delay when the positivity of the error signals is specified. Also, the observer matrices can be obtained through the solution of LMIs, which can be easily checked by using standard software packages.

The rest of this paper is organized as follows. Section II gives some notations and preliminaries. In Section III, we first present some equivalent conditions to characterize the stability of positive time-delay systems, and further treat the synthesis problem of positive observers for interval PLDT systems with time delay. In Section IV, a numerical example is provided to demonstrate the efficacy of the proposed approach. Finally, we summarize our results in Section V.

\section{PRELIMINARIES AND PROBLEM Formulation}

Notation: Let $\mathbb{N}$ be the set of nonnegative integers; $\mathbb{R}$ is the set of real numbers; $\mathbb{R}^{n}$ denotes the $n$-column vectors; $\mathbb{R}^{m \times n}$ is the set of all real matrices of dimension $m \times n . \mathbb{R}_{+}^{m \times n}$ represents the $m \times n$ dimensional matrices with nonnegative components and $\mathbb{R}_{+}^{n} \triangleq \mathbb{R}_{+}^{1 \times n}$. For a matrix $A \in \mathbb{R}^{m \times n}$, $a_{i j}$ denotes the element located at the $i$ th row and the $j$ th column. Matrix $A$ is said to be nonnegative if $\forall(i, j) a_{i j} \geq 0$; it is said to be positive, if $\forall(i, j) a_{i j} \geq 0, \exists(i, j) a_{i j}>0$. Considering the fact that the definitions of nonnegative and positive matrices are equivalent, except when a nonnegative matrix is identically zero which is the degenerate case and is of no interest, we do not distinguish these two throughout this paper. That is to say that we consider these two are equivalent in general cases. $A$ is said to be strictly positive, denoted as $A>>0$, if $\forall(i, j) a_{i j}>0$. For matrices $A$, $B \in \mathbb{R}^{m \times n}$, the notation $A \geq \geq B$ (respectively, $A>>B$ ) means that $A-B$ is positive (respectively, strictly positive). For matrices $A, A_{m}, A_{M} \in \mathbb{R}^{m \times n}$, the notation $A \in$ $\left[A_{m}, A_{M}\right]$ means that $A_{m} \leq \leq A \leq \leq A_{M}$. For any real symmetric matrices $P, Q$, the notation $P \succeq Q$ (respectively, $P \succ Q$ ) means that the matrix $P-Q$ is positive semi-definite (respectively, positive definite). For any matrix $A \in \mathbb{R}^{n \times n}$, $\rho(A) \triangleq \max \{|\lambda|: \lambda \in \sigma(A)\}$ denotes the spectral radius of $A$, where $\sigma(A)$ is the spectrum of $A$. $A$ is said to be Schur stable if and only if $\rho(A)<1$.
$I$ and 0 represent identity matrix and zero matrix respectively; $\operatorname{diag}(\ldots)$ stands for a block-diagonal matrix. The superscript " $T$ " denotes matrix transpose and the symbol \# is used to represent a matrix which can be inferred by symmetry. Matrices, if their dimensions are not explicitly stated, are assumed to be with compatible dimensions for algebraic operations.

\section{A. Preliminaries}

Consider the following linear discrete-time system with time delay

$$
\left\{\begin{aligned}
x(k+1) & =A x(k)+A_{d} x(k-d)+B u(k), \\
y(k) & =C x(k)+C_{d} x(k-d), \\
x(\theta) & =\varphi(\theta), \theta=-d,-d+1, \ldots, 0
\end{aligned}\right.
$$

where $x(k) \in \mathbb{R}^{n}$ is the state vector, $u(k) \in \mathbb{R}^{p}$ is the input, $y(k) \in \mathbb{R}^{q}$ is the output or measurement, respectively; $A, A_{d}$, $B, C$, and $C_{d}$ are real constant matrices with appropriate dimensions. $d \in \mathbb{N}$ is constant, and $\varphi(\theta)$ is the initial condition. When there is no time delay in (1), that is, $A_{d}=0$, we recover the usual linear discrete-time system

$$
\left\{\begin{aligned}
x(k+1) & =A x(k)+B u(k), \\
y(k) & =C x(k), x(0)=x_{0} .
\end{aligned}\right.
$$

Next, we recall some definitions and characterizations of positive linear systems.

Definition 1: A linear dynamic system in the form of (2) is said to be positive, if for every $x_{0} \in \mathbb{R}_{+}^{n}$ and every $u(k) \in$ $\mathbb{R}_{+}^{p}$, we have $x(k) \in \mathbb{R}_{+}^{n}, y(k) \in \mathbb{R}_{+}^{q}$ for $k \geq 0$.

Definition 2: A linear dynamic system in the form of (1) is said to be positive, if for every $\varphi(\theta) \in \mathbb{R}_{+}^{n}(\theta=-d,-d+$ $1, \ldots, 0)$ and every $u(k) \in \mathbb{R}_{+}^{p}$, we have $x(k) \in \mathbb{R}_{+}^{n}, y(k) \in$ $\mathbb{R}_{+}^{q}$ for $k \geq 0$.

The following two lemmas show that one can verify whether a discrete-time system is positive or not by simply checking the sign of system matrices involved in the mathematical model of the corresponding system.

Lemma 1 ([2]): System (2) is positive if and only if $A, B$, and $C$ are positive matrices.

Lemma 2 ([7]): System (1) is positive if and only if $A$, $A_{d}, B, C$, and $C_{d}$ are positive matrices.

To facilitate the subsequent analysis, a characterization on the stability of positive linear discrete-time systems (2) is introduced as follows:

Lemma 3 ([2]): The positive linear discrete-time systems in (2) with $u(t)=0$ is asymptotically stable if and only if there exists a matrix $P=\operatorname{diag}\left(p_{1}, p_{2}, \ldots, p_{n}\right) \succ 0$ such that

$$
A^{T} P A-P \prec 0 .
$$

\section{B. Problem Formulation}

Consider the following positive interval system with time delay:

$$
\left\{\begin{aligned}
x(k+1) & =A x(k)+A_{d} x(k-d), \\
y(k) & =C x(k)+C_{d} x(k-d), \\
x(\theta) & =\varphi(\theta), \theta=-d,-d+1, \ldots, 0 .
\end{aligned}\right.
$$


Here, the system matrices $A, A_{d}, B, C$, and $C_{d}$ are not known but belong to the uncertainty set

$$
\begin{array}{r}
\mathfrak{S}=\left\{\left(A, A_{d}, C, C_{d}\right): A \in\left[A_{m}, A_{M}\right], A_{d} \in\left[A_{d m}, A_{d M}\right],\right. \\
\left.C \in\left[C_{m}, C_{M}\right], C_{\tau} \in\left[C_{d m}, C_{d M}\right]\right\}
\end{array}
$$

where $A_{m} \in \mathbb{R}_{+}^{n \times n}, A_{d m} \in \mathbb{R}_{+}^{n \times n}, C_{m} \in \mathbb{R}_{+}^{q \times n}, C_{d m} \in$ $\mathbb{R}_{+}^{q \times n}$.

In this paper, we aim at estimating the system state $x(k)$ in (3). To be more specific, we are interested in constructing the following general observer:

$$
\hat{x}(k+1)=\hat{A} \hat{x}(k)+\hat{B} y(k),
$$

where $\hat{x}(k) \in \mathbb{R}^{n}$ for $k \geq 0$. $\hat{A}$ and $\hat{B}$ are observer matrices to be determined.

Let $e(k)=x(k)-\hat{x}(k)$, then the augmented system of (3) and (4) can be described by

$$
\xi(k+1)=\mathcal{A} \xi(k)+\mathcal{A}_{d} \xi(k-d)
$$

where $\xi(k)=\left[x^{T}(k), e^{T}(k)\right]^{T}$, and

$$
\mathcal{A}=\left[\begin{array}{cc}
A & 0 \\
A-\hat{A}-\hat{B} C & \hat{A}
\end{array}\right], \mathcal{A}_{d}=\left[\begin{array}{cc}
A_{d} & 0 \\
A_{d}-\hat{B} C_{d} & 0
\end{array}\right] .
$$

As far as the state observation problem is concerned, it is well known that, for general linear time-delay system, to design an observer in (4) is equivalent to require that (5) is asymptotically stable. However, such a specification may not be enough for the positive systems (3), since it is highly desirable that the estimate $\hat{x}(k)$ should be positive, like the signal $x(k)$ to be estimated. Thus, to ensure the positivity of $\hat{x}(k)$, it is natural to require that the observer (4) should be a positive system, which is equivalent to require, according to Lemma 1 , matrices $\hat{A}$ and $\hat{B}$ in (4) are positive. In addition, when there is no time delay in the original system (3), it can be seen from [6] that the induced error signal is positive for the identity Luenberger observer case. Thus, it is hoped that such a specification should be also preserved for the general observer in (4). In fact, the positivity of signal $e(k)$ is embedded for the consideration of both consistency with the case of identical observer and synthesis convenience. Based on the above discussion, we formulate the positive observer problem in this paper as follows.

Problem POD (Positive Observer Design): Given a positive system with both parameter uncertainties and time delay in (3), design a positive observer in the form of (4) such that the augmented system in (5) is positive and asymptotically stable for any $\left(A, A_{d}, C, C_{d}\right) \in \mathfrak{S}$, that is, the following two requirements should be satisfied simultaneously:

- System (5) is positive and asymptotically stable for any $\left(A, A_{d}, C, C_{d}\right) \in \mathfrak{S}$;

- Observer matrices $\hat{A}$ and $\hat{B}$ are positive.

Remark 1: In the observer (4), the Schur stability of matrix $\hat{A}$ is a prerequisite for the augmented system (5) to be asymptotically stable. In addition, since the observer (4) cannot affect the system state of (3), the stability of $x(k)$ then is a necessary condition for the asymptotic stability of $\xi(k)$.

\section{Main Results}

The problem to be discussed in this section is to synthesize the positive observer for the positive systems with both interval parameter uncertainties and time delay. To achieve this, we first present equivalent conditions on the asymptotic stability of positive systems (3), and then consider the observer synthesis problem in the sequence. In particular, we will show that observer matrices can be constructed through the solutions of a set of LMIs, which is dependent upon the time delay when the positivity of error signals is considered.

\section{A. Stability of PLDT Systems with Time Delay}

In this subsection, the objective is to give conditions on the global asymptotic stability of positive time-delayed systems in (3), which play an essential role in the synthesis of positive observers.

Theorem 1: Given positive system (3), the following statements are equivalent:

(i) System (3) is asymptotically stable for any $d \in \mathbb{N}$.

(ii) The following positive system

$$
x(k+1)=\left(A+A_{d}\right) x(k)
$$

is asymptotically stable.

(iii) Matrix $A+A_{d}$ is Schur stable, that is, $\rho\left(A+A_{d}\right)<1$.

(iv) There exists a positive diagonal matrix $P \succ 0$ satisfying

$$
\left(A+A_{d}\right)^{T} P\left(A+A_{d}\right)-P \prec 0 .
$$

(v) There exists a positive diagonal matrix $P \succ 0$ satisfying

$$
\left[\begin{array}{cc}
-P & \left(A+A_{d}\right)^{T} P \\
\# & -P
\end{array}\right] \prec 0 .
$$

(vi) There exist positive diagonal matrices $P_{0} \succ 0$ and $P_{n} \succ$ 0 such that

$$
\left[\begin{array}{cc}
A^{T} P_{0} A+P_{n}-P_{0} & A^{T} P_{0} A_{d} \\
\# & A_{d}^{T} P_{0} A_{d}-P_{n}
\end{array}\right] \prec 0 .
$$

Proof: We prove the equivalence of these statements by the following line.

(ii) $\Longleftrightarrow$ (iii) The equivalence of (ii) and (iii) can be obtained immediately form the definition of Schur stability.

(iv) $\Longleftrightarrow(v)$ Based upon Schur complement, the equivalence of (iv) and (v) is obtained immediately.

(i) $\Longrightarrow$ (ii) It follows from (i) that (6) is asymptotically stable if we let $d=0$ in system (3).

(ii) $\Longrightarrow$ (iv) Since (6) is a positive system, according to Lemma 3, we have the conclusion in (iv).

(iv) $\Longrightarrow$ (i) It follows from (7) that the following system

$$
y(k+1)=\left(A+A_{d}\right)^{T} y(k)
$$

is positive and asymptotically stable, thus, $\rho\left(\left(A+A_{d}\right)^{T}\right)<$ 1. Then, choose $y(0)>>0$ such that

$$
p \triangleq \sum_{k=1}^{\infty} y(k)>>0 .
$$


Moreover, from (10), we have

$$
\begin{aligned}
\sum_{k=1}^{\infty} y(k) & =\sum_{k=0}^{\infty}\left[\left(A+A_{d}\right)^{T}\right]^{k} y(0) \\
& =\left[I-\left(A+A_{d}\right)^{T}\right]^{-1} y(0) .
\end{aligned}
$$

Let $r \triangleq y(0)>>0$, we further obtain

$$
p=\left[I-\left(A+A_{d}\right)^{T}\right]^{-1} r
$$

or equivalently,

$$
\left[I-\left(A+A_{d}\right)^{T}\right] p=r>>0 .
$$

Choose the Lyapunov functional

$$
V(k)=p^{T} x(k)+\sum_{l=1}^{d} p^{T} A_{d} x(k-l) .
$$

Calculating the difference of $V(k)$ along the solution of system (3), one has

$$
\begin{aligned}
& V(k+1)-V(k) \\
= & p^{T}\left(A x(k)+A_{d} x(k-d)\right)-p^{T} x(k) \\
& +p^{T} A_{d} x(k)-p^{T} A_{d} x(k-d) \\
= & -p^{T}\left[I-\left(A+A_{d}\right)\right] x(k),
\end{aligned}
$$

which together with (12) yields that $V(k+1)-V(k)<$ 0 . Then it follows from standard Lyapunov theory that the positive time-delay system in (3) is asymptotically stable.

(vi) $\Longleftrightarrow$ (i) If we define

$$
\times(k)=\left[x^{T}(k), x^{T}(k-1), \ldots, x^{T}(k-d)\right]^{T},
$$

then system (3) can be described equivalently as follows.

$$
\mathrm{x}(k+1)=\mathrm{A} \times(k)
$$

where

$$
\mathrm{A}=\left[\begin{array}{ccccc}
A & 0 & \cdots & 0 & A_{d} \\
I & 0 & \cdots & 0 & 0 \\
\vdots & \vdots & \ddots & \vdots & \vdots \\
0 & 0 & \cdots & 0 & 0 \\
0 & 0 & \cdots & I & 0
\end{array}\right]
$$

From Lemma 3, we have that system (3) is asymptotically stable, if and only if there exists a diagonal matrix $\mathrm{P}=$ $\operatorname{diag}\left(P_{0}, P_{1}, \ldots, P_{n}\right) \succ 0$ such that $\mathrm{A}^{T} \mathrm{PA}-\mathrm{P} \prec 0$. After some mathematical manipulation, we have condition (9). For details, we refer readers to [15]. This completes the whole proof.

Remark 2: From the above theorem, one can see that the size of time delay does not affect the asymptotic stability of positive linear discrete-time systems [7], [15]. Moreover, from (iii) and the inequality $\rho\left(A+A_{d}\right) \geq$ $\max \left\{\rho(A), \rho\left(A_{d}\right)\right\}$, we can further conclude that matrices $A$ and $A_{d}$ are both Schur stable.

Remark 3: It can be easily seen that conditions (iv), (v), and (vi) are all expressed in the form of linear matrix inequalities, which can be easily verified by existing software. Different from those in [7] and [13], where the stability criteria are given in the form of vector inequalities and characteristic polynomial, respectively, the present LMItype characterization will greatly facilitate the synthesis of positive observers to be considered in the sequel. On the other hand, note that these three conditions are equivalent among each other and delay-independent, with the number of decision variables in (iv) and (v) less than the one in (vi), which may further indicate that conditions (iv) and (v) may perform more effectively in the sense of numerical computation.

\section{B. Positive Observer for Interval PLDT Systems with Time Delay}

In this subsection, we shall focus on the synthesis of positive observers for interval PLDT systems with time delay. We establish a necessary and sufficient condition to guarantee the positivity and asymptotic stability of the augmented system in (5) for any $\left(A, A_{d}, C, C_{d}\right) \in \mathfrak{S}$, and the observer matrices are expressed explicitly through the solution of LMIs.

Theorem 2: Problem POD is solvable if and only if there exist diagonal matrices $P=\operatorname{diag}\left(p_{1}, p_{2}, \ldots, p_{n}\right) \succ 0, Q=$ $\operatorname{diag}\left(q_{1}, q_{2}, \ldots, q_{n}\right) \succ 0$, and positive matrices $L \geq \geq 0$, $G \geq \geq 0$ such that the following LMIs hold:

$$
\begin{aligned}
& \Xi \triangleq\left[\begin{array}{cccc}
-P & 0 & \Xi_{13} & \Xi_{14} \\
\# & -Q & 0 & L^{T} \\
\# & \# & -P & 0 \\
\# & \# & \# & -Q
\end{array}\right] \prec 0, \\
& Q A_{m}-L-G C_{M} \geq \geq 0 \\
& Q A_{d m}-G C_{d M} \geq \geq 0
\end{aligned}
$$

where

$$
\begin{aligned}
& \Xi_{13}=\left(A_{M}+A_{d M}\right)^{T} P, \\
& \Xi_{14}=\left(A_{M}+A_{d M}\right)^{T} Q-L^{T}-\left(C_{m}+C_{d m}\right)^{T} G^{T} .
\end{aligned}
$$

In this case, a desired observer in (4) can be obtained with

$$
\hat{A}=Q^{-1} L, \hat{B}=Q^{-1} G \text {. }
$$

Proof: (Sufficiency) When (15)-(18) are satisfied, we first show that the augmented system (5) is positive. To this end, we note from (16)-(18) that

$$
\left\{\begin{array}{c}
Q A_{m}-Q \hat{A}-Q \hat{B} C_{M} \geq \geq 0, \\
Q A_{d m}-Q \hat{B} C_{d M} \geq \geq 0 .
\end{array}\right.
$$

Note $Q \succ 0$ is diagonal, we have

$$
\left\{\begin{array}{c}
A_{m}-\hat{A}-\hat{B} C_{M} \geq \geq 0, \\
A_{d m}-\hat{B} C_{d M} \geq \geq 0 .
\end{array}\right.
$$

Observe that for any $\left(A, A_{d}, C, C_{d}\right) \in \mathfrak{S}$, we obtain

$$
\left\{\begin{array}{l}
A \geq \geq A_{m}, A_{d} \geq \geq A_{d m}, \\
\hat{B} C_{M} \geq \geq \hat{B} C, \hat{B} C_{d M} \geq \geq \hat{B} C_{d} .
\end{array}\right.
$$

This together with (20) yields that

$$
\left\{\begin{array}{c}
A-\hat{A}-\hat{B} C \geq \geq 0, \\
A_{d}-\hat{B} C_{d} \geq \geq 0,
\end{array}\right.
$$


which further implies that $\mathcal{A} \geq \geq 0$, and $\mathcal{A}_{d} \geq \geq 0$ in system (5). Then, based on Lemma 2, we conclude that system (5) is positive. In the following, we will show that system (5) is asymptotically stable for any $\left(A, A_{d}, C, C_{d}\right) \in \mathfrak{S}$.

From (15) and (18), we have

$$
\tilde{\Xi} \triangleq\left[\begin{array}{cccc}
-P & 0 & \left(A_{M}+A_{d M}\right)^{T} P & \tilde{\Xi}_{14} \\
\# & -Q & 0 & \hat{A}^{T} Q \\
\# & \# & -P & 0 \\
\# & \# & \# & -Q
\end{array}\right] \prec 0
$$

where

$$
\tilde{\Xi}_{14}=\left(\left(A_{M}+A_{d M}\right)-\hat{A}-\hat{B}\left(C_{m}+C_{d m}\right)\right)^{T} Q .
$$

Based on Schur complement, (23) is equivalent to

$$
\begin{aligned}
& {\left[\begin{array}{cc}
A_{M}+A_{d M} & 0 \\
A_{M}+A_{d M}-\hat{A}-\hat{B}\left(C_{m}+C_{d m}\right) & \hat{A}
\end{array}\right]^{T}} \\
& \times\left[\begin{array}{cc}
P & 0 \\
0 & Q
\end{array}\right] \\
& \times\left[\begin{array}{cc} 
& \\
A_{M}+A_{d M}-\hat{A}-\hat{B}\left(C_{m}+C_{d m}\right) & \hat{A}
\end{array}\right] \\
& -\left[\begin{array}{cc}
P & 0 \\
0 & Q
\end{array}\right]
\end{aligned}
$$$$
\prec 0 \text {. }
$$

Note that $P \succ 0$ and $Q \succ 0$, we have

$$
\rho\left(\left[\begin{array}{cc}
A_{M}+A_{d M} & 0 \\
A_{M}+A_{d M}-\hat{A}-\hat{B}\left(C_{m}+C_{d m}\right) & \hat{A}
\end{array}\right]\right)<1 .
$$

On the other hand, for any $\left(A, A_{d}, C, C_{d}\right) \in \mathfrak{S}$, it is obvious that

$$
\begin{aligned}
0 & \leq \leq \mathcal{A}+\mathcal{A}_{d} \\
& \leq \leq\left[\begin{array}{cc}
A_{M}+A_{d M} & 0 \\
A_{M}+A_{d M}-\hat{A}-\hat{B}\left(C_{m}+C_{d m}\right) & \hat{A}
\end{array}\right]
\end{aligned}
$$

Then, considering (25) and (26), it can be deduced that

$$
\rho\left(\mathcal{A}+\mathcal{A}_{d}\right)<1 .
$$

Therefore, by Theorem 1, we have that the positive delay system (5) is asymptotically stable.

(Necessity) If there exist matrices $\hat{A}$ and $\hat{B}$ such that system (5) is positive and asymptotically stable for any $\left(A, A_{d}, C, C_{d}\right) \in \mathfrak{S}$, then we have

$$
\begin{aligned}
{\left[\begin{array}{cc}
A_{m} & 0 \\
A_{m}-\hat{A}-\hat{B} C_{M} & \hat{A}
\end{array}\right] } & \geq \geq 0, \\
{\left[\begin{array}{cc}
A_{d m} & 0 \\
A_{d m}-\hat{B} C_{d M} & 0
\end{array}\right] } & \geq \geq 0 .
\end{aligned}
$$

These two inequalities directly imply that

$$
\left\{\begin{array}{c}
A_{m}-\hat{A}-\hat{B} C_{M} \geq \geq 0 \\
A_{d m}-\hat{B} C_{d M} \geq \geq 0 .
\end{array}\right.
$$

Considering the fact that $Q \succ 0$ is diagonal, we have

$$
\left\{\begin{array}{c}
Q A_{m}-Q \hat{A}-Q \hat{B} C_{M} \geq \geq 0, \\
Q A_{d m}-Q \hat{B} C_{d M} \geq \geq 0 .
\end{array}\right.
$$

By setting $L \triangleq Q \hat{A}, G \triangleq Q \hat{B}$, we are ready to obtain, from the diagonal positivity of $Q$, that $L \geq \geq 0, G \geq \geq 0$. Then it follows from (27) that (16) and (17) hold.

In addition, since the positive delay system (5) is asymptotically stable, from Theorem 1 , we obtain that there exist matrices $P=\operatorname{diag}\left(p_{1}, p_{2}, \ldots, p_{n}\right) \succ 0, Q=$ $\operatorname{diag}\left(q_{1}, q_{2}, \ldots, q_{n}\right) \succ 0$ such that

$$
\left[\begin{array}{cccc}
-P & 0 & \left(A_{M}+A_{d M}\right)^{T} P & \tilde{\Xi}_{14} \\
\# & -Q & 0 & \hat{A}^{T} Q \\
\# & \# & -P & 0 \\
\# & \# & \# & -Q
\end{array}\right] \prec 0,
$$

where $\tilde{\Xi}_{14}$ is defined in (24). Note that $\hat{A}^{T} Q=L^{T}$ and $\hat{B}^{T} Q=G^{T}$, we further obtain (15). This completes the whole proof.

Remark 4: It should be emphasized that the introduction of parameter uncertainties destroy some nice properties, such as the identical Luenberger approach and structural decomposition techniques, which was broadly adopted previously, can no longer be applicable. In Theorem 2, we develop a necessary and sufficient condition to design positive observers for positive systems in the presence of interval parameter uncertainties and time delay, which is effective for the case when a priori knowledge about the size of time delay $d$ in (3) is not available.

Remark 5: The obtained results in this paper can be easily extended to the case where positive linear discrete-time systems (3) are with multiple time delays, since one may use the approach in [13] (Chap. 8, Page 423) to formulate the positive delayed system to be an augmented positive system. Then, by applying the same idea developed in the proof of Theorem 2, we can derive the analogous results readily.

\section{Numerical Simulation}

In this section, we present an example to demonstrate the applicability of the proposed approach.

Consider a positive linear discrete-time system in (3) with parameters as follows:

$$
\begin{aligned}
& A_{m}=\left[\begin{array}{ll}
0.2681 & 0.2576 \\
0.0728 & 0.3455
\end{array}\right], C_{m}=\left[\begin{array}{ll}
1 & 0
\end{array}\right], \\
& A_{d m}=\left[\begin{array}{ll}
0.1892 & 0.1756 \\
0.0000 & 0.1145
\end{array}\right], C_{d m}=\left[\begin{array}{ll}
0 & 0
\end{array}\right] \text {, } \\
& A_{M}=\left[\begin{array}{ll}
0.2810 & 0.3012 \\
0.0788 & 0.3520
\end{array}\right], C_{M}=\left[\begin{array}{ll}
1.1 & 0
\end{array}\right] \text {, } \\
& A_{d M}=\left[\begin{array}{ll}
0.2014 & 0.1860 \\
0.0000 & 0.1145
\end{array}\right], C_{d M}=\left[\begin{array}{ll}
0 & 0
\end{array}\right] \text {. }
\end{aligned}
$$

It can be verified that the eigenvalue of matrix $A_{M}+A_{d M}$ is $\lambda_{1}=0.6764$ and $\lambda_{2}=0.2846$, which further indicates that, based on Theorem 1, this positive system is asymptotically stable. The aim of this example is to design a general observer in the form of (4) such that the augmented system 
is positive and asymptotically stable. By virtue of standard software, we obtain the feasible solution of (15)-(17) as follows:

$$
\begin{aligned}
& P=\left[\begin{array}{ll}
0.9975 & 0.0000 \\
0.0000 & 1.9736
\end{array}\right], Q=\left[\begin{array}{ll}
1.7024 & 0.0000 \\
0.0000 & 2.6842
\end{array}\right], \\
& L=\left[\begin{array}{ll}
0.1802 & 0.1094 \\
0.1094 & 0.6083
\end{array}\right], G=\left[\begin{array}{l}
0.1503 \\
0.0396
\end{array}\right]
\end{aligned}
$$

Thus, from (18), the desired observer matrices are given as

$$
\hat{A}=\left[\begin{array}{ll}
0.1059 & 0.0643 \\
0.0408 & 0.2266
\end{array}\right], \hat{B}=\left[\begin{array}{l}
0.0883 \\
0.0148
\end{array}\right],
$$

that is,

$$
\begin{aligned}
& \left\{\hat{x}_{1}(k+1)=0.1059 \hat{x}_{1}(k)+0.0643 \hat{x}_{2}(k)+0.0883 y(k),\right. \\
& \left\{\hat{x}_{2}(k+1)=0.0408 \hat{x}_{1}(k)+0.2266 \hat{x}_{2}(k)+0.0148 y(k)\right. \text {. }
\end{aligned}
$$

\section{CONCLUSiON}

In this paper, the problem of positive observer design for positive linear discrete-time systems with both interval parameter uncertainties and time delay has been investigated. Necessary and sufficient conditions are established to ensure the asymptotic stability of positive systems with time delay, which are expressed under the framework of linear matrix inequalities. In addition, an easily verifiable condition is developed to study the existence of positive observers for interval PLDT systems with time delay, and the observer matrices can be obtained through the solution of a set of LMIs, which can be easily computed by means of standard software. The effectiveness of the derived condition has been demonstrated by a numerical example.

\section{REFERENCES}

[1] A. Berman and R. J. Plemmons, Nonnegative Matrices in the Mathematical Sciences. Philadephia, Pa: SIAM, 1994.

[2] L. Farina and S. Rinaldi, Positive Linear Systems: Theory and Applications. Wiley-Interscience, 2000.

[3] V. G. Rumchev and D. J. G. James, "Controllability of positive linear discrete-time systems," Int. J. Control, vol. 50, no. 3, pp. 845-857, 1989.

[4] N. K. Son and D. Hinrichsen, "Robust Stability of positive continuous time systems," Numerical Functional Analysis and Optimization, vol. 17, no. 5, pp. 649-659, 1996.

[5] B. D. O. Anderson, M. Deistler, L. Farina, and L. Benvenuti, "Nonnegative realization of a linear system with nonnegative impulse response," IEEE Trans. Circuits and Systems (I), vol. 43, no. 2, pp. 134-142, Feb. 1996.

[6] J. M. van den Hof, "Positive linear observers for linear compartmental systems," SIAM J. Control Optim., vol. 36, no. 2, pp. 590-608, Mar. 1998.

[7] W. M. Haddad and V. S. Chellaboina, "Stability theory for nonnegative and compartmental dynamical systems with time delay," Systems \& Control Letters, vol. 51, no. 5, pp. 355-361, 2004.

[8] L. Benvenuti and L. Farina, "A tutorial on the positive realization problem,” IEEE Trans. Automat. Control, vol. 49, no. 5, pp. 651-664, May. 2004.

[9] E. Fornasini and M. E. Valcher, "Controllability and reachability of 2D positive systems: a graph theoretic approach," IEEE Trans. Circuits and Systems (I), vol. 52, no. 3, pp. 576-585, Mar. 2005.

[10] H. Gao, J. Lam, C. Wang, and S. Xu, "Control for stability and positivity: equivalent conditions and computation," IEEE Trans. Circuits and Systems (II), vol. 52, no. 9, pp. 540-544, Sep. 2005.

[11] M. A. Rami and F. Tadeo, "Controller synthesis for positive linear systems with bounded controls," IEEE Trans. Circuits and Systems (II), vol. 54, no. 2, pp. 151-155, Feb. 2007.
[12] J. Back and A. Astolfi, "Design of positive linear observers for positive linear systems via coordinate transformations and positive realizations," SIAM J. Control Optim., vol. 47, no. 1, pp. 345-373, Jan. 2008.

[13] T. Kaczorek, Polynomial and Rational Matrices: Applications in Dynamical Systems Theory. London: Springer-Verlag, 2007.

[14] Z. Shu, J. Lam, H. Gao, B. Du, and L. Wu, "Positive observers and dynamic output-feedback controllers for interval positive linear systems," IEEE Trans. Circuits and Systems (I), vol. 55, no. 10, pp. 3209-3222, Nov. 2008.

[15] L. Wu, J. Lam, Z. Shu, and B. Du, "On stability and stabilizability of positive delay systems," Asian Journal of Control, vol. 11, no. 2, pp. 226-234, 2009.

[16] M. A. Rami, U. Helmke, and F. Tadeo, "Positive observation problem for linear time-delay positive systems," in 2007 Mediterranean Conference on Control and Automation, Jul. 2007, pp. T19-027. 